\title{
40 GHz Metamaterial Development Using Hybrid Electromagnetic/Statistical Tools
}

\author{
Daniela Staiculescu, Lara Martin, Manos Tentzeris \\ Georgia Institute of Technology \\ Atlanta, GA, 30332, U.S.A. \\ daniela@ece.gatech.edu
}

\begin{abstract}
The successful use of Design of Experiments (DOE) and Path of Ascent (POA) approaches in conjunction with time-domain full wave commercial simulators in a feasibility and design study of a metamaterial structure is presented. The frequency of interest is $40 \mathrm{GHz}$ and the technology used is multilayer Low Temperature Cofired Ceramic (LTCC). The chosen approach for the Double Negative Metamaterial implementation is a loaded Coplanar Waveguide (CPW) transmission line. The design goals are a resonant frequency of $40 \mathrm{GHz}$ and minimum insertion loss at that frequency. The electromagnetic performance of the loaded transmission line is simulated with a full wave time domain commercial simulator. The results of these simulations are incorporated into the DOE and POA techniques. First, the significant factors in achieving each of the goals are identified, then statistical models are developed for the two output variables and applied to optimize the structure.
\end{abstract}

Keywords: optimization, metamaterials, TLM simulators, statistical, design of experiments, path of ascent.

\section{Introduction}

The concept of metamaterials, or left-handed materials (LHM), double negative (DNG), negative refractive index (NRI) materials, has been around since the late 1960's [1], but only recently has it gained a lot of interest since practical implementation solutions emerged. These materials exhibit phase and group velocities of opposite signs and a negative refractive index in certain frequency ranges, both characteristics making them desirable for RF and microwave applications. One of the implementation approaches starts from the equivalent transmission line model and artificially loads a host line with a dual periodical structure of series capacitors and shunt inductors [2]-[3], as shown in Fig. 1.

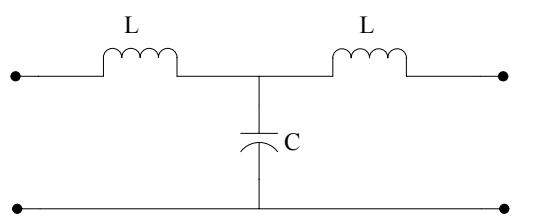

(a)

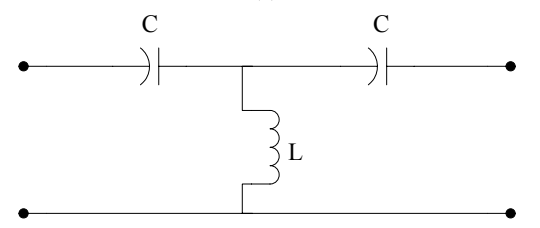

(b)

Fig. 1. Transmission line model (a) conventional (b) dual.

The length of the period and the value of the capacitors and inductors determine the frequency band in which the material has DNG behavior. One of the main challenges for the high frequency implementation, where the dimension become smaller and the process design rules become very restrictive, is the choice of the inductor and capacitor geometry to obtain the required left-handed passband and minimum insertion loss at the desired operating frequency. The goal of this work is to design and optimize, for the first time, a metamaterial 
structure for $40 \mathrm{GHz}$. The current design methods do not take into account the specific effect of each of the factors involved in the design process and the degree these factors interact with each other. The use of DOE and POA allows these goals to be achieved.

\section{Statistical background}

A design of experiments is a series of tests in which a set of input variables or factors is purposely changed so that the experimenter can observe and identify the reasons for changes in the output response. Previous work shows the use of design of experiments in modeling of $\mathrm{RF} /$ microwave circuits [4]. The factorial designs are used in experiments involving several factors where the goal is the study of the joint effects of the factors on a response. Prior knowledge of the analyzed system is required for choosing the factors and their studied ranges. The $2^{k}$ factorial design is the simplest one, with $k$ factors at 2 levels each. It provides the smallest number of runs for studying $k$ factors and is widely used in factor screening experiments [5]. Center points in the DOE increase the capability of investigating the model fit, including curvature in the response, and account for variation in the fabrication process of the structure. The DOE data is analyzed and then POA is applied to determine a path, which extends beyond the DOE design space, for further optimization of the figures of merit. Using this path, another design space is defined and the process repeats with another full factorial DOE with center points. The process is complete when the performance goal or optimum performance is achieved.

This paper shows the first use of the DOE and POA in a study of metamaterial design for frequencies as high as $40 \mathrm{GHz}$, saving a lot of time and frustration compared to the traditional trial-and-error approach.

\section{Metamaterial structure analysis and design}

The proposed structure is implemented in Low Temperature Cofired Ceramic (LTCC) technology, and the host transmission line is a $75 \Omega$ coplanar waveguide (CPW). The advantage of the CPW is the ease to build shunt lumped elements due to the availability of the ground plane on the same layer as the signal, eliminating the need for vias. The series capacitors and shunt inductors are implemented as shown in Fig. 2.

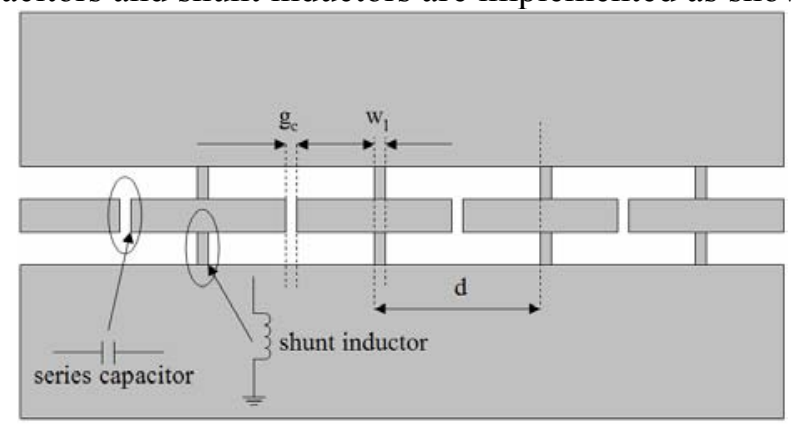

Fig. 2. CPW implementation of metamaterial structure.

The preliminary analysis of the structure dispersion diagram showed that the factors that influence the system performance the most are the values of the series capacitors and shunt inductors, as well as the length of the period. The experiment has been set for a constant characteristic impedance of $75 \Omega$, so the width of the signal line and the gap for the CPW are kept constant. Therefore, the three variables in the experiment are the gap of the capacitor $g_{c}$, the width of the inductive line $w_{l}$, and the length of the period $d$. A full factorial experiment with three factors consists of $2^{3}=8$ treatment combinations. The two levels and the center point values chosen for each input variable have been controlled by the fabrication process and are presented in Table 1 .

\begin{tabular}{|c|c|c|c|}
\hline Variable & Min $(\boldsymbol{\mu m})$ & Max $(\boldsymbol{\mu m})$ & Center Point $(\boldsymbol{\mu m})$ \\
\hline $\boldsymbol{D}$ & 1760 & 1840 & 1800 \\
\hline $\boldsymbol{g}_{\boldsymbol{c}}$ & 130 & 170 & 150 \\
\hline $\boldsymbol{w}_{\boldsymbol{l}}$ & 90 & 110 & 100 \\
\hline
\end{tabular}

Table1. Variables for the $2^{3}$ experiment 
The output variables are the resonant frequency in the first LH passband $f_{\text {res }}$ and the value of the insertion loss at that frequency $I L$. The eight simulations have been run in MicroStripes TLM Modeler and then inputed in a statistical analysis software.

The initial DOE revealed the significant parameters for both figures of merit and then first-order prediction models were developed based on them. At the $95 \%$ confidence level, it was found that all three parameters $d, g_{c}$, and $w_{l}$ were statistically significant for the insertion loss and only $g_{c}$ and $d$ were significant for the resonant frequency $f_{\text {res }}$. Additionally, the model for insertion loss $I L$ was validated for model assumptions of normality and equal variance of the residuals. The model for the resonating frequency $f_{\text {res }}$ was validated for normality assumption [6], but there appeared to be curvature due to the center point residuals, when the equal variance assumption was investigated. No lack of fit for either model was confirmed at the $99 \%$ confidence level.

Based on the first-order prediction model for insertion loss $I L$, the POA was determined for optimization of insertion loss, while the predicted resonant frequency was monitored. The fourth step of the POA was found to be optimum, based on decreased insertion loss and constraint of design rules for the input variables.

Based on these POA results, the fourth step became the center point of the second DOE and ranges that were proportional to the first DOE were used for the experimental design. At the $95 \%$ confidence level, it was found that all three parameters $d, g_{c}$, and $w_{l}$ and the interaction of $w_{l}$ and $d$ were statistically significant for insertion loss IL. At the $95 \%$ confidence level, it was found that all three parameters $d$, $g_{c}$, and $w_{l}$, the interaction of $g_{c}$ and $d$, and the interaction of $w_{l}$ and $d$ were statistically significant for resonant frequency $f_{\text {res }}$. Both models were validated for normality assumption, but there appeared to be a slight funnel of residuals, increasing with increasing response, when the equal variance assumption was investigated. A slight lack of fit for both models was confirmed at the $99 \%$ confidence level. The models are given by the following equations:

$$
\begin{aligned}
& I L=5.88-0.166\left(\frac{d-1875}{40}\right)+0.531\left(\frac{g_{c}-90}{20}\right) \\
& +0.339\left(\frac{w_{l}-81}{10}\right)+0.206\left(\frac{w_{l}-81}{10}\right)\left(\frac{d-1875}{40}\right)
\end{aligned}
$$

$$
\begin{aligned}
& f_{\text {res }}=40.0-0.224\left(\frac{d-1875}{40}\right)+0.216\left(\frac{g_{c}-90}{20}\right) \\
& +0.131\left(\frac{w_{l}-81}{10}\right)+0.234\left(\frac{g_{c}-90}{20}\right)\left(\frac{d-1875}{40}\right) \\
& +0.234\left(\frac{w_{l}-81}{10}\right)\left(\frac{d-1875}{40}\right)
\end{aligned}
$$

Before accepting (1)-(2), the models were confirmed. Confirmations of the models were performed for the following combination of parameters: $d=1896 \mu \mathrm{m}, g_{c}=96 \mu \mathrm{m}$, and $w_{l}=84 \mu \mathrm{m}$. This configuration was simulated in the electromagnetic simulator and was also predicted with the developed models. The results of the simulation, compared to the DOE $95 \%$ confidence intervals defined by the lower and upper bounds for the predicted resonant frequency $f_{\text {res }}$ and insertion loss $I L$ are shown in Table 1.

\begin{tabular}{|c|c|c|}
\hline & $\boldsymbol{f}_{\text {res }}[\mathrm{GHz}]$ & $\boldsymbol{I L}[\mathrm{dB}]$ \\
\hline Simulation & 39.93 & 5.918 \\
\hline DOE lower bound & 39.72 & 5.598 \\
\hline DOE upper bound & 40.43 & 6.582 \\
\hline
\end{tabular}

Table 1. Resonant frequency and insertion loss from electromagnetic simulation compared to the DOE 95\% confidence intervals

Because the simulation values fall into the $95 \%$ confidence intervals from the DOE, the DOE models were confirmed and accepted as the final models for optimization.

\section{Model interpretation and optimization}

The models offer the option to optimize performance with respect to either figure of merit or both simultaneously. They can also be used to predict the performance of the system for a specific configuration. In 
this case, the optimization goals were minimized insertion loss $I L$ for a target resonant frequency of $40 \mathrm{GHz}$. Error! Reference source not found. shows the surfaces of possible solutions for the optimization goals. The optimization is done based on the intersection of the surfaces, which is shown in Error! Reference source not found. The values that satisfied the optimization conditions within the design space of the second DOE were $d$ $=1915 \mu \mathrm{m}, g_{c}=75 \mu \mathrm{m}$, and $w_{l}=75 \mu \mathrm{m}$ leading to the optimized values of the two figures of merit of $I L=4.99$ and $f_{\text {res }}=39.2 \mathrm{GHz}$. Figure 1 shows a plot of the simulated optimized metamaterial structure performance.

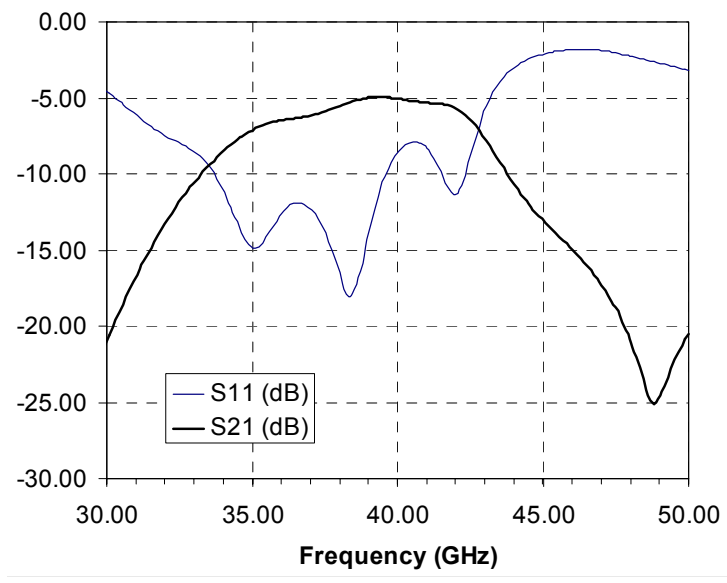

Figure 1. Performance of optimized structure

\section{Conclusions}

This paper presents a method in which deterministic electromagnetic simulation tools and statistical modeling methods can be used to optimize RF components and microsystems. To prove the concept, a benchmarking geometry of a metamaterial structure in LTCC technology was chosen. The two optimized responses were the center frequency and the insertion loss. Three geometric parameters of the structure were chosen as experimental factors. The results of the hybrid electromagnetic-statistical analysis generated statistical models that could be used to predict the filter performance based on the geometry of the structure. These models could then be used to optimize the filter with respect to desired performance, enabling the system-level optimization of the geometry in quick and inexpensive way. In this case, the center frequency was chosen and the insertion loss was minimized to exemplify the possibilities of the method.

The proposed approach can be easily extended to a larger number of design variables and optimized figures of merit. The advantage of the POA approach is that the method provides direction for further optimization, which may extend beyond the initial DOE design space, and is able to distinguish local performance maxima from paths of continued performance optimization. In this way, the behavior of a complex system, such as a 3D multilayer module, could be predicted at the beginning of the design process, leading to a much shorter design cycle of added functions, while achieving design optimization goals in a simple and elegant manner.

\section{References}

[1] V. G. Veselago, "The electrodynamics of substances with simultaneously negative values of $\varepsilon$ and $\mu$ ", Sov. Phys. Usp., vol. 10, no.4, pp. 509-514, Jan. - Feb. 1968

[2] G. V. Eleftheriades, A. K. Iyer, P. C. Kremer, "Planar Negative Refractive Index Media Using Periodically L-C Loaded Transmission Lines", IEEE Trans. Microwave Theory \& Tech.,vol. 50, no. 12, pp. 2702-2712, December 2002.

[3]A. Grbic, G. V. Eleftheriades, "Experimental Verification of Backward-Wave Radiation from a Negative Refractive Index Material", Journal of App. Physics, vol. 92, no. 10, pp. 5930-5935, November 2002

[4] D.Staiculescu, J.Laskar, E.M.Tentzeris, "Design Rule Development for Microwave Flip-Chip Applications", IEEE Trans. Microwave Theory \& Tech., Vol.48, No.9, pp.1476-1481, September 2000.

[5] D.C. Montgomery, Design and Analysis of Experiments, New York: J. Wiley \& Sons, 1997.

[6] A. Dean et al, Design and Analysis of Experiments, Springer-Verlag New York, Inc., New York, 1999 